\title{
Durability of Wireless Networks of Battery-Powered Devices
}

\author{
Maryam Soltan and Massoud Pedram \\ University of Southern California \\ Dept. of Electrical Engineering \\ Los Angeles, CA 90089 \\ \{soltan,pedram\}@usc.edu
}

\begin{abstract}
Given the criticality of energy awareness in wireless networks, it has become essential to devise an improved definition of the network lifetime at the system design stage. The new definition must capture the life profile of the network while accounting for its functionality and specific design parameters. This paper presents the notion of network durability, which captures the spatiotemporal life/death patterns of devices in a wireless network by examining the time evolution of spatial patterns according to which devices are progressively forced to exit the network having exhausted their energy resource. Using network durability, we show how networks can satisfy different levels of monitoring criticality, even when they exhibit the same conventionally defined lifetime. Finally, as an example application, we consider a heterogeneous location-aware modulation scheme where the proposed durability model is effectively employed to characterize the network lifetime.
\end{abstract}

Keywords: Sensor Networks; Wireless Ad Hoc and Sensor Devices; Lifetime; Battery-powered Devices; Energy-awareness.

\section{INTRODUCTION}

In a wireless network a large number of battery operated devices gather, process, and transmit information. Given the initial energy level for every device, the Quality of Service (QoS) and lifetime are typically considered as the two main criteria in evaluating the efficacy of a network design and the underlying protocols. Both criteria are functions of different parameters such as the density and location of devices, network connectivity, communication request rate, and cost. Typically, the spatial coverage is crucial to the system. Local failures in a region can lead to degradation in QoS. Hence, the duration of time that the service is available depends on the aggregated longevity of devices, which is in turn dictated by their initial energy along with energy depletion rates.

Much research has focused on energy/lifetime aware design of wireless networks [1-13]. Various definitions of lifetime, proposed in the literature to date, are mostly generic and do not account for the specifics of node locations, communication characteristics, network connectivity, and local coverage. For example, many researchers have tried to maximize the network lifetime, considering a single node failure or a given percentage of dead nodes as indicators for the end of network lifetime. A survey of different lifetime definitions is presented in [1].
Given a desired function for a network, the network lifetime can generally be defined as the duration of time from its deployment until loss of functionality [2]. Examples include a certain fraction of dead nodes, coverage loss, or connectivity loss. In many applications, percentage of dead nodes alone [1][2] cannot determine the system longevity, unless actual locations and specific impacts of dead nodes on the overall QoS are taken into account. In [2] lifetime is defined as the cumulative active time of the network until the first loss of coverage or QoS failure. In [3] lifetime is studied based on the energy for a network with multi-hop routing, by considering distance-based summation of the lifetimes. Without knowledge about individual devices, (e.g., their locations and communication), these definitions typically fail to accurately account for the impact of a design decision on the overall energy efficiency of the network. Little attention has been paid to address the effect of the livelihood, i.e., life quality, and longevity of each individual device on the overall spatiotemporal network behavior.

To improve the practicality of the lifetime as a criterion at the design stage, a deeper understanding of life pattern of the network is necessary. Clearly, precise definition of the relationship between the network lifetime and lifetime of devices in an application-specific manner is an important design task. For example, in some applications, the network may tolerate a large percentage of dead devices as long as they are in specific locations or perform non-critical functions.

We present a novel methodology for looking at lifetime of the network by incorporating the time evolution of the spatial profile of alive/dead nodes. We introduce the concept of network durability and present a model for it. In doing so, we examine the spatial life/death profile in the network over time. We quantify the effect of the battery depletion rates and locations of the devices on the overall network durability.

The broader impact of this work is to assist in the analysis and evaluation of different designs and protocols for energy-constrained networks. We use a location-aware modulation scheme [4] to show how our approach can be used to evaluate energy-awareness of different system design strategies. 
In Section II, we present system description and models. Network durability and life and death contours are presented in Section III. In Section IV, we discuss location-aware modulation scheme as an application example. Simulation results are presented in Section $\mathrm{V}$, and concluding remarks are provided in the last section.

\section{Motivation AND Models}

Our motivation is to devise a more efficient methodology that can be used in different applications to study and quantify how various design decisions can affect the energyefficiency and battery usage of the network. Such a methodology either can be used at the design time to decide on the network organization, topology and detailed layout or can be utilized to devise lifetime-aware network protocols. We define energy depletion state as the durability metric for the network. Using an example application, we show how our network durability metric can quantify the energy efficiency and energy balancing of the network. We first review modulation-aware mechanisms. Then we describe the system specification followed by energy consumption model.

\section{A. Modulation-Aware Techniques}

Modulation scaling and adaptive modulation techniques are presented in [11]-[13]. In these techniques, one common modulation scheme is considered and the target bit error rate is adjusted to achieve lower energy per bit. Another approach is to consider a heterogeneous location-aware modulation scheme [4] where different nodes may use different modulation schemes under the same bit error rate constraint. We focus on this latter approach, and show how selection of the modulation schemes for different devices can affect the life and death profile and ultimately network durability.

\section{B. System Description}

Hierarchical networks reduce the communication burden on devices and hence increase the network lifetime [6-9]. An example is a two-tiered network where relay nodes with higher energy resources relay data from the end devices to a gateway. We consider a network consisting of clusters of event aggregator relays (EAR) and low power devices [8]. EARs with higher communication capabilities and higher energy source, collect local data, aggregate it, and send it towards the gateway. We consider a deterministic multipleaccess approach based on a combined Frequency Division Multiple Access (FDMA) and Time Division Multiple Access (TDMA) scheme to avoid any co-channel interference.

In such a hierarchical network, livelihood depends on the life of each cluster, and hence on the energy consumption of each device. We focus on the effect of device failure in a cluster on the lifetime. The life of a cluster is ended when associated devices cannot perform the required functionality, which means that the QoS in some parts is lower than a minimum required level (we call it the critical level).

\section{Energy Consumption Model}

Energy dissipation due to data transmission is a large percentage of the overall energy consumption. Using a logdistance path loss model [14], the required energy per transmitted bit in the $i^{\text {th }}$ device is

$$
e_{T x}(i)=k \cdot E_{b} \cdot\left(d_{e(i), i}\right)^{\beta_{e(i), i}}
$$

where $k$ is a constant, which depends on channel bandwidth, antenna gain, amplifier efficiency, and Noise Figure of the receiver. $E_{b}$ is the desired energy per bit at the receiver in order to satisfy the bit error rate (BER) requirement. $d_{e(i), i}$ and $\beta_{e(i), i}$ respectively are the distance and the path loss exponent between node $i$ and its assigned EAR.

For any modulation scheme, BER can be characterized as a function of $E_{b} / N_{0}$ which is the ratio of energy per bit over noise power spectral density [15]. Due to bit errors, there is a possibility for un-successful transmission. Let $B E P$ denote the bit error probability. For a packet of length $L$ the probability of successful transmission from node $i$ is

$$
\operatorname{prob}_{i}=(1-B E P)^{L}
$$

By re-transmission of a packet until successful delivery, the success probability would be a geometric random variable and hence the expected number of trials to succeed is $1 /$ prob $_{i}$. Therefore, the average energy consumption in node $i$ for successfully transmitting a packet to the EAR is

$$
e_{\text {packet }}(i)=\frac{L \cdot e_{T x}(i)}{\text { prob }_{i}}
$$

Note that our methodology remains agnostic to these assumptions and can be extended to more general models.

\section{LIFE PROFILE AND NETWORK DURABILITY}

A device is dead when it does not have sufficient energy to transmit even one data packet. Network lifetime clearly depends on the lifetimes of individual devices. However, the exact relationship may not be straightforward. For now, we may informally define network durability as the duration of time that the network meets the QoS constraints and remains connected (see below for the precise characterization of the network durability). Devices communicate directly to a designated EAR, so the QoS in a cluster depends on coverage and quality of communication links. If a region of the network satisfies the required regional criticality level, we call it a functional region. Failure of some nodes due to depleted batteries can cause the network to be less useful, but the level of damage may not reach a critical level. In other words, the same percentage of loss with different distribution of dead nodes may result in operational network or one which is useless (out of service) depending on the spatial distribution and criticality of the lost devices. Actual 
location and responsibility of any node in the network defines the level of damage (loss of functionality) to the network once that node drops out of the network due to lack of energy.

We will discuss a more precise definition of network durability but first let's define the notion of Blackout in a network, which is borrowed from the electric power system literature. Regional Blackout is power loss due to death of all devices in a region. Based on the size of the region, and the effects of the regional nodes on the coverage of a larger area and connectivity of the network, a blackout becomes a widespread blackout that terminates the functionality of the network either by connectivity loss or coverage loss. Based on these definitions, we define the network durability i.e., the duration of time that the network remains generally functional and connected, as the time to first widespread blackout. Indeed what determines the real service lifetime and energy-awareness of a network is the durability of the network and not the overall percentage of dead nodes. So in this paper whenever we refer to longevity, and lifetime, we specifically consider the durability of the network. The network is considered dead (has reached the end of its service life) only when a widespread blackout occurs. In the following subsections we discuss life and death contours and spatial energy depletion state as the durability metric.

\section{A. Life and Death (LaD) Contours, Durability Profile and Energy Depletion State (ENDS)}

To quantify the network durability, we look at time evolution of Life and Death (LaD) contours and we define ENergy Depletion State (ENDS). LaD contours at any time are utilized to provide information about the spatial distribution of alive/dead devices. In dead contours all nodes are out of battery and regional blackout occurs.

Let's define ENergy Depletion State (ENDS) metric based on the normalized energy consumption of a device with respect to its initial energy. Hence, for node $i$ at time $t$, $\operatorname{ENDS}(i, t)$ is defined as follows:

$$
\operatorname{ENDS}(i, t)=\frac{P_{c} . t+e_{\text {packet }}(i) \cdot N_{t x}(i, t)}{E_{\text {Battery }}(i)}
$$

where $N_{t x}(i, t)$ is the average number of instantiated packets by node $i$ until time $t$. $E_{\text {Battery }}(i)$ is the initial battery energy, $e_{\text {packet }}(i)$ denotes average energy per packet transmission, and $P_{c}$ is the average maintenance power consumption in the node.

$E N D S$ metric demonstrates the age of the node, which is zero when its battery is completely full and increases to 1 as its battery energy is exhausted. For a node, ENDS shows how fast the energy source is being exhausted, while its spatial gradient over the nodes across the network shows how the remaining battery source varies over the covered area.

Consider a cluster $j$ with $n_{R j}$ nodes distributed in a region, $R_{j}$. $E N D S_{c l}\left(R_{j}, t\right)$ denotes the spatial average of energy depletion in this cluster at time $t$ as the weighted average of
ENDS of individual nodes in the cluster at that time.

$$
\operatorname{ENDS}_{c l}\left(R_{j}, t\right)=\frac{1}{n_{R_{j}}} \sum_{i \in R_{j}} s w(i, t) \cdot \operatorname{ENDS}(i, t)
$$

where $s w(i, t)$ denotes the significance weight of node $i$ at time $t$. This weight reflects the importance of the device in maintaining the QoS, i.e., a user specified device priority, and the intra-cluster connectivity (or per our definition of durability, in stopping a small - possibly pointwiseblackout from spreading out to the rest of the cluster). We consider $E N D S_{c l}$ as network durability metric and use it to calculate the expected cluster durability values. More precisely, the larger the ENDS value of a cluster, the shorter its expected durability. In this model we allow the application to change the weights of the nodes over time based on the mission criticality, which may in turn change over time e.g., a device may become critical for stopping a cluster-wide blackout after a number of its neighbors leave the cluster. In general, the significance weight $s w(i, t)$ may be calculated by the EAR based on the LaD contour information. If we assume that the QoS is correlated with the spatial density of alive devices in the cluster, then we can define the operational density $o d_{c l}\left(R_{j}, t\right)$ of each cluster $j$ based on the number of alive nodes $\left(n_{\text {alive }}\left(R_{j}, t\right)\right)$ in the cluster at time $t$ and total number of nodes in the cluster $\left(n_{R j}\right)$, as follows:

$$
\operatorname{od}_{c l}\left(R_{j}, t\right)=\frac{n_{\text {alive }}\left(R_{j}, t\right)}{n_{R_{j}}}
$$

And finally the significant weight of any alive node $i$ in region $R_{j}$ at time $t$ is,

$$
\forall i_{\text {alive }} \in R_{j}, \quad s w\left(i_{\text {alive }}, t\right)=\left(\frac{1}{o d_{c l}\left(R_{j}, t\right)}\right)^{\alpha} \text { with } \alpha \geq 1
$$

For each region, we define durability at time $t$ as the remaining time to a first regional or cluster-wide blackout and calculate it as follows:

$$
D u r_{c l}\left(R_{j}, t\right)=\frac{1-E N D S_{c l}\left(R_{j}, t\right)}{\left(\partial E N D S_{c l}\left(R_{j}, t\right) / \partial t\right)}
$$

The above definitions can be applied recursively up the levels of a network. For example, in a network comprising of $m$ clusters or regions with $s w_{c l}\left(R_{j}, t\right)$ denoting the significance weight of the cluster $j$ in the overall network at time $t$, we have:

$$
\begin{gathered}
\operatorname{ENDS}_{\text {net }}(t)=\frac{1}{m} \sum_{j \in n e t}\left[s w_{c l}\left(R_{j}, t\right) \cdot E N D S_{c l}\left(R_{j}, t\right)\right] \\
D u r_{n e t}(t)=\frac{1-E N D S_{n e t}(t)}{\frac{1}{m} \sum_{j \in n e t}\left[s w_{c l}\left(R_{j}, t\right) \cdot\left(\partial E N D S_{c l}\left(R_{j}, t\right) / \partial t\right)\right]}
\end{gathered}
$$

with $s w\left(R_{j}, t\right)$ defined and calculated based on the notion of operational density of cluster heads or sub regions in the overall network. The network durability thus represents the remaining time to widespread blackout. 


\section{SAMPlE APPLiCATION SCENARIOS}

For this paper as an application scenario, we consider a two-tier wireless sensor network. In such a network, when all the sensors in part of a cluster die, a regional blackout occurs. In our simulation studies we show that a small local blackout in a cluster can change over time into a wide spread blackout. To study the life behavior of a network, we shall utilize the LaD contours and ENDS durability metrics. We study two different scenarios. In the first scenario we do not employ any optimizations for energy efficiency or lifetime extension, and we consider homogeneous modulation assignment for all the nodes within a cluster. In the second scenario, we consider a location-aware heterogeneous modulation scheme.

In location-aware heterogeneous modulation scheme [4], energy consumption distribution within a cluster is balanced by using various modulation schemes for different nodes within a cluster. Let's consider sensors randomly distributed in a circular area of radius $R$ where the EAR is located at the center of the region. For the sensors which are closer to the EAR, Modulation 1 is selected as a more bandwidth efficient modulation scheme that requires larger $E_{b} / N_{0}$ for a fixed BER. However, for those sensors that are farther away, Modulation 2 is selected as the less bandwidth efficient modulation scheme that can achieve the same BER with smaller $E_{b} / N_{0}$. As an example, let's consider Differential Quadrature Phase Shift Keying (DQPSK) as Modulation 1 for the sensors within distance $r$ from the EAR, and coherent Binary phase Shift Keying (BPSK) as Modulation 2 for the remaining sensors. If Modulation 1 requires $E_{b}$ Jules per bit for a given BER of 1e-4, Modulation 2 will achieve the same BER with about $0.55 E_{b}$ Jules per bit [16]. Although Modulation 2 is less bandwidth efficient, utilizing it for the nodes with larger distance to the EAR, and therefore require higher transmission energy, can result in better spatial balancing of life in the sensors within the cluster and by extension across the network.

Larger number of diverse modulation schemes may be employed in different regions of a cluster in order to achieve more balanced remaining energy level distribution as the network ages. In this application scenario we can calculate $E N D S_{R 1}, E N D S_{R 2}$, and $E N D S_{c l}$ respectively for regions with Modulation 1, 2, and the cluster. Lower value of $E N D S_{c l}$ specifies a network with higher durability.

\section{SiMULATIONS}

We present an experimental study to evaluate the efficacy of the proposed durability model. We look at spatial values of $E N D S$ parameter over time. In our results, the energy consumption and the number of transmitted packets over time are obtained from packet-level simulation of the network and are used to obtain ENDS values. We describe system setup and then explain the simulation procedures and results.

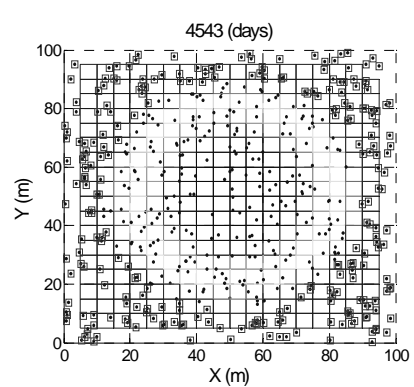

(a)

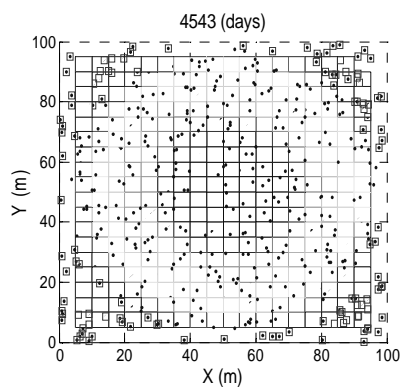

(b)
Figure 1: Snapshot of Life and Death (LaD) contours with square nodes denoting dead devices in a cluster.

(a) Homogeneous modulations. (b) Heterogeneous modulations.

\section{A. System Setup}

We randomly placed 500 sensors in 100x100 $\mathrm{m}^{2}$ field for each cluster with EAR located in the center. Due to inherent symmetry, the results for one cluster are representative for the overall network. Packet size is set to 128 bytes, while the average sensing rate is set to 0.6 packets per sec. The initial energy level of each sensor is set to $2 \mathrm{~kJ}$ and the path loss exponent is set to 3 . We consider modulation schemes BPSK, and DQPSK. To achieve 0.01\% BER, assuming additive white Gaussian noise channels, the $E_{b} / N_{0}$ for these modulations are 8.4, and $11 \mathrm{~dB}$, respectively [16].

\section{B. Simulation Procedure}

We consider enough FDMA/TDMA channels to avoid any co-channel interference. Also to reduce interference from neighboring clusters, different frequency bands are assigned to the neighboring clusters. The bit rate for sensorto-EAR links is set to 40 and $80 \mathrm{kbps}$ for sensors with BPSK and DQPSK modulations respectively. Two scenarios are considered, (I) homogeneous modulation scheme with DQPSK modulation for all the sensors, and (II) locationaware heterogeneous modulation scheme with DQPSK and BPSK modulations. DQPSK is used for the centrally located sensors within distance of $r$ from their EAR and BPSK is assigned to the other sensors. Simulations are done for different values of $r$.

\section{Simulation Results}

Figure 1 shows the node distribution and LaD contours within one cluster for homogeneous and heterogeneous modulation schemes after 4,543 days. Middle dots represent live sensors and the squares represent the dead ones. In the homogeneous case $50 \%$ of the sensors are dead, while in the heterogeneous modulation scheme only $24 \%$ of the sensors are dead. Time evolution of $\mathrm{LaD}$ shows regional blackouts. Figure 2 represents variation of spatial average of $E N D S_{c l}$ over time. In this graph at any time ENDS of homogeneous scheme has a larger value, which signifies that the lifetime of the network using this scheme will ends sooner than the 


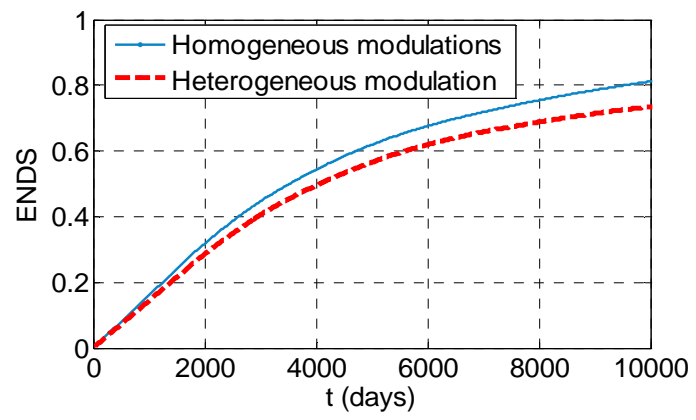

Figure 2: temporal variation of $E N D S_{c l}$

heterogeneous case.

Figures 3 and 4 show the spatial distribution of ENDS values at different simulation times. Figure 3 is based on the simulation Scenario I. Simulation times correspond to the events where $2 \%, 10 \%$, and $50 \%$ of sensors run out of battery. Results in Figure 4 are based on scenario II with $r$ equal to 30 meters. These results are reported for exactly the same simulation times as in Figure 3 and show improved spatial balancing on ENDS and lower spatial average, and hence improved durability metric. Lower values of ENDS indicate longer durability of the network. Table I reports lifetime of the network for various values for $r$ according to a generic definition of lifetime based on different
Table I: Lifetime (Days) according to definition of lifetime based on different percentage of dead nodes.

\begin{tabular}{|c|c|c|c|c|c|}
\hline & $\mathrm{r}=10$ & $\mathrm{r}=20$ & $\mathrm{r}=30$ & $\mathrm{r}=40$ & $\mathrm{r}=50$ \\
\hline $2 \%$ dead & 1886 & 1886 & 1886 & 1886 & 1886 \\
\hline $5 \%$ dead & 2421 & 2419 & 2403 & 2394 & 2183 \\
\hline $10 \%$ dead & 2904 & 2902 & 2900 & 2897 & 2461 \\
\hline $20 \%$ dead & 4070 & 4069 & 4066 & 4062 & 2782 \\
\hline
\end{tabular}

percentages of dead nodes. These values show how a generic definition for lifetime fails to correctly capture the effect of $r$, as a design parameter. On the other hand, Figure 5 shows the spatial distribution of ENDS for the simulation Scenario II at the time that $2 \%$ of the sensors are dead, clearly highlighting the impact of changing $r$. In Figure 5(c) average $E N D S$ for the centrally located sensors is much higher than the other two cases. Figure 6 shows the variation of spatial average of $E N D S_{c l}$ with respect to different values of $r$ for the simulation Scenario II at the time that $2 \%$ of the sensors are dead. Note that, larger values of $r$ correspond to cases where higher numbers of nodes communicate using a less energy-efficient modulation scheme. The results clearly show that as $r$ becomes larger, the ENDS metric increases, which means that the network functionality ends sooner.

\section{CONCLUDING REMARKS}

We introduced the concept of network durability as a

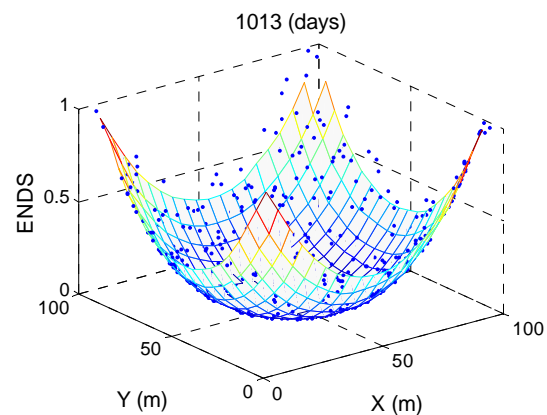

(a)

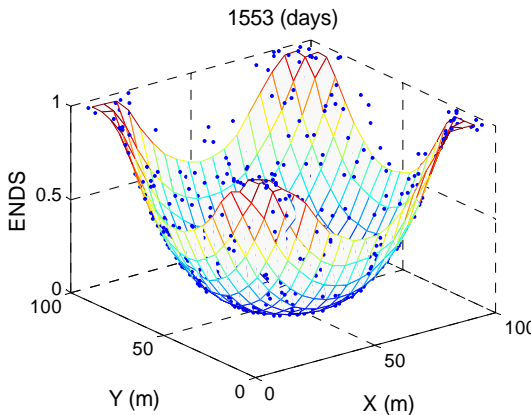

(b)

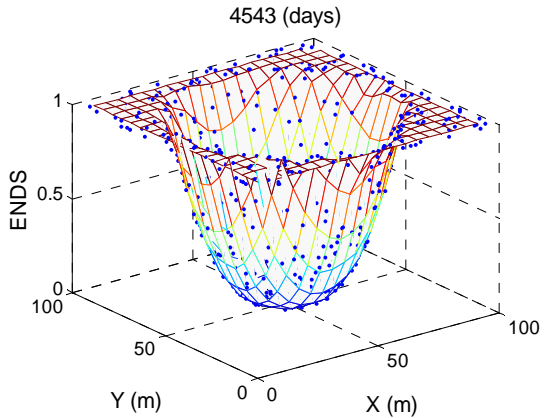

(c)

Figure 3: Spatial values of ENDS with homogeneous modulation assignments at different simulation times.

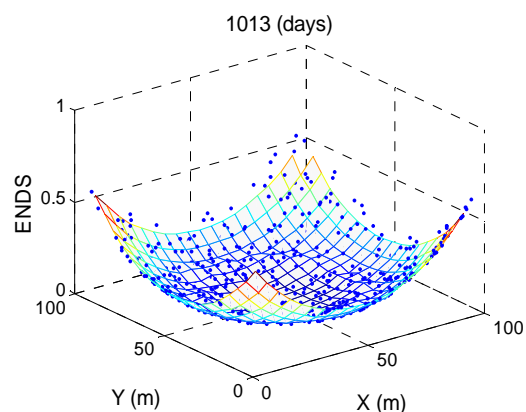

(a)

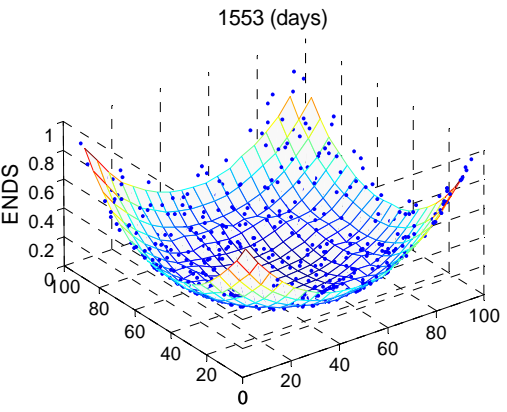

(b)

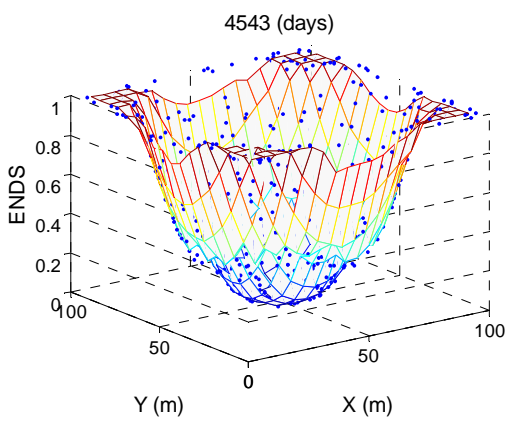

(c)

Figure 4: Spatial values of $E N D S$ with heterogeneous modulation scheme at different simulation times 


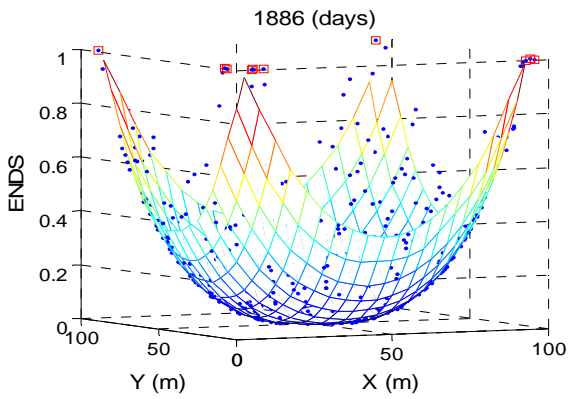

(a)

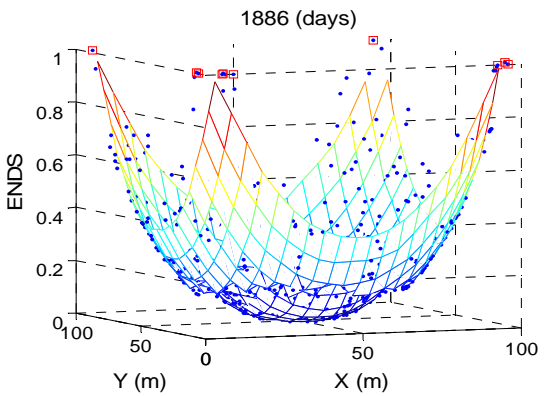

(b)

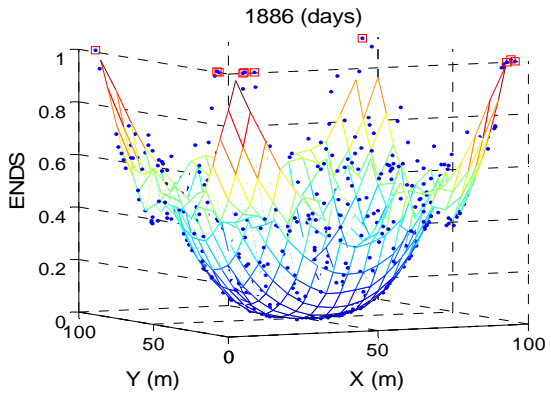

(c)

Figure 5: Heterogeneous modulation scheme when $2 \%$ of the sensors are dead (at time 1886 days). Spatial distribution of ENDS for (a) $r=10$ meters, (b) $r=30$ meters, and (c) $r=45$ meters.

quantitative spatiotemporal measure of the lifetime, which accounts for the impact of specific system parameters in lifetime. We introduced ENDS as a durability metric incorporating energy balancing. We proposed a new insight into temporal/spatial pattern of life and death in the network and looked at the dying snapshots of the network. Moreover, we studied the effect of positioning of the devices, and their life degradation rate on the overall durability metrics. Based on these insights and metrics, we quantified the network durability. We used a heterogeneous location-aware modulation scheme in hierarchical sensor network as an application example where our durability model can be deployed. We studied its implications on the dying pattern of the network, and showed how heterogeneous modulation can improve network durability without necessarily impacting the initial percentage of sensors that die first. This example clearly demonstrates how a network durability model can offer better insight into the livelihood of a network when compared to more classical definitions of lifetime.

\section{REFERENCES}

[1] Q. Dong, "Maximizing system lifetime in wireless sensor networks," IPSN'05, April 2005.

[2] A. Swami, Q. Zhao, Y.-W. Hong, and L. Tong, Wireless Sensor Networks Signal Processing and Communications Perspectives, John Wiley \& Sons, 2007.

[3] K. Sha, W. Shi, "Modeling the lifetime of wireless sensor network," American Scientific Publishers, Sensor Letters, Vol. 3, 2005.

[4] M. Soltan, I. Hwang, and M. "Heterogeneous Modulation for Tradingoff Energy Balancing with Bandwidth Efficiency in Hierarchical Sensor Networks,” Proc. of Int'l Symposium on a World of Wireless, Mobile and Multimedia Networks, Jun. 2008.

[5] Santashil PalChaudhuri, Rajnish Kumar, Richard Baraniuk, David B. Johnson, "Design of adaptive overlays for multi-scale communication in sensor networks, International Conference on Distributed Computing in Sensor Systems, June 2005.

[6] V. P. Mhatre, C. Rosenberg, D, Kofman, R. Mazumdar, and N. Shroff, "A minimum cost heterogeneous sensor network with lifetime constraint," IEEE Transactions on Mobile Computing, Vol. 4, No1, pp. 4-15, 2005.

[7] A. Iranli, M. Maleki, and M. Pedram, "Energy-efficient strategies for

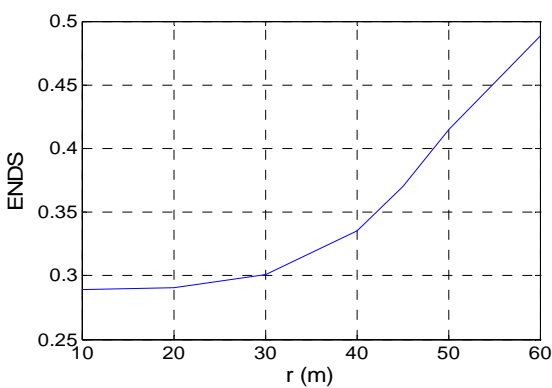

Figure 6: Spatial average of ENDS $\left(\right.$ ENDS $\left._{\mathrm{cl}}\right)$ vs. $r$ in the scenario II with heterogeneous modulation scheme when $2 \%$ of the sensors are dead (at time 1886 days).

deployment of a two-level wireless sensor network," Proc. of SLPED, Aug. 2005, pp. 233-238.

[8] M. Soltan, M. Maleki, and M. Pedram, "Lifetime-aware hierarchical wireless sensor network architecture with mobile overlays," Proc. of IEEE Radio and Wireless Symposium, pp. 325-328, Jan. 2007.

[9] V. Raghunathan, C. Schurgers, S. Park, and M. Srivastava, "EnergyAware wireless microsensor networks," IEEE Signal Processing Magazine, vol. 19, pp. 40-50, March 2002.

[10] H. kwon, T. H. Kim, S. Choi, and B. G. Lee, "A cross-layer strategy for energy-efficient reliable delivery in wireless sensor networks," IEEE Transactions on Wireless Communications, vol. 5, no. 12, pp. 36893699, 2006.

[11] C. Schurgers, O. Aberborne, and M. Srivastava, "Modulation scaling for energy-aware communication systems," Proc. of ISLPED, pp. 9699, 2001.

[12] Z. Yang, Y. Yuan, J. He, and W. Chen, "Adaptive modulation scaling scheme for wireless sensor networks," IEICE Transactions on Communications, pp. 882-889, 2005.

[13] J. J. E. Garzás, C. B. Calzón, and G Armada, “An Energy-Efficient Adaptive Modulation Suitable for Wireless Sensor Networks with SER and Throughput Constraints," EURASIP Journal on Wireless Communications and Networking, 2007.

[14] Rapparport, T. S. , Wireless Communications, Principles and Practice, Prentice Hall, 1996.

[15] J. G Proakis., Digital Communications, McGraw-Hill Inc., Third Edition, 1995.

[16] F. Xiong, Digital Modulation Techniques, Artech House, 2000. 